\title{
Nonexistence results for semi-linear Moore-Gibson-Thompson equation with nonlocal operator
}

\author{
Ali Hakem ${ }^{a}$, Svetlin Georgiev ${ }^{b}$ \\ ${ }^{a}$ Laboratory ACEDP, Djillali Liabes university, 22000 Sidi Bel Abbes, Algeria. \\ ${ }^{b}$ Faculty of Mathematics and Informatics, Sofia University, Sofia, Bulgaria.
}

\begin{abstract}
We study the nonexistence of global weak solutions to the following semi-linear Moore - Gibson- Thompson equation with the nonlinearity of derivative type, namely,

$$
\begin{cases}u_{t t t}+u_{t t}-\Delta u-(-\Delta)^{\frac{\alpha}{2}} u_{t}=\left|u_{t}\right|^{p}, & x \in \mathbb{R}^{n}, \quad t>0, \\ u(0, x)=u_{0}(x), \quad u_{t}(0, x)=u_{1}(x), & u_{t t}(0, x)=u_{2}(x) \quad x \in \mathbb{R}^{n}\end{cases}
$$

where $\alpha \in(0,2], \quad p>1$, and $(-\Delta)^{\frac{\alpha}{2}}$ is the fractional Laplacian operator of order $\frac{\alpha}{2}$. Then, this result is extended to the case of a weakly coupled system. We intend to apply the method of a modified test function to establish nonexistence results and to overcome some difficulties as well caused by the well-known fractional Laplacian $(-\Delta)^{\frac{\alpha}{2}}$.The results obtained in this paper extend several contributions in this field.
\end{abstract}

Keywords: Test functions nonexistenc lifespan estimates.

2010 MSC: 35B33, 35B44, 35R11, 35L76.

\section{Introduction}

The main goal of this paper is to discuss the nonexistence of global weak solutions to the following semi-linear Moore-Gibson-Thompson equation

$$
\begin{cases}u_{t t t}+u_{t t}-\Delta u-(-\Delta)^{\frac{\alpha}{2}} u_{t}=\left|u_{t}\right|^{p}, \quad x \in \mathbb{R}^{n}, \quad t>0, \\ u(0, x)=u_{0}(x), \quad u_{t}(0, x)=u_{1}(x), \quad u_{t t}(0, x)=u_{2}(x), \quad x \in \mathbb{R}^{n}\end{cases}
$$

Email addresses: hakemali@yahoo.com (Ali Hakem), svetlingeorgiev1@gmail.com (Svetlin Georgiev) 
where $p>1, n \geq 1, \alpha \in(0,2]$, and $(-\Delta)^{\frac{\alpha}{2}}$ is the fractional Laplacian operator of order $\frac{\alpha}{2}$. We extend our analysis to the case of a weakly coupled system, more precisely,

$$
\left\{\begin{array}{l}
u_{t t t}+u_{t t}-\Delta u-(-\Delta)^{\frac{\alpha}{2}} u_{t}=\left|v_{t}\right|^{p}, \quad x \in \mathbb{R}^{n}, \quad t>0, \\
v_{t t t}+v_{t t}-\Delta v-(-\Delta)^{\frac{\beta}{2}} v_{t}=\left|u_{t}\right|^{q}, \quad x \in \mathbb{R}^{n}, \quad t>0, \\
u(0, x)=u_{0}(x), \quad u_{t}(0, x)=u_{1}(x), \quad u_{t t}(0, x)=u_{2}(x), \quad x \in \mathbb{R}^{n}, \\
v(0, x)=v_{0}(x), v_{t}(0, x)=v_{1}(x), v_{t t}(0, x)=v_{2}(x), \quad x \in \mathbb{R}^{n} .
\end{array}\right.
$$

Recently, the nonexistence of global (in time) solutions to the following system

$$
\left\{\begin{array}{l}
u_{t t}-\Delta u+(-\Delta)^{\delta_{1}} u_{t}=|v|^{p}, \quad x \in \mathbb{R}^{n}, \quad t>0 \\
v_{t t}-\Delta v+(-\Delta)^{\delta_{2}} v_{t}=|u|^{q}, \quad x \in \mathbb{R}^{n}, \quad t>0 \\
u(0, x)=u_{0}(x), \quad u_{t}(0, x)=u_{1}(x), \quad x \in \mathbb{R}^{n} \\
v(0, x)=v_{0}(x), \quad v_{t}(0, x)=v_{1}(x) \quad x \in \mathbb{R}^{n}
\end{array}\right.
$$

is investigated in [10]. It was shown that if $\delta_{1}, \delta_{2} \in\left[0, \frac{1}{2}\right], u_{0}=u_{1}=0$ and $u_{1}, v_{1} \in \mathbb{L}^{1}\left(\mathbb{R}^{n}\right)$ satisfy

$$
\int_{\mathrm{IR}^{n}} u_{1}(x) d x>\varepsilon_{1}, \quad \int_{\mathrm{IR}^{n}} u_{1}(x) d x>\varepsilon_{2}
$$

and

$$
\begin{aligned}
& \frac{n}{2} \leq \frac{1+q \frac{1-\delta_{2}}{1-\delta_{1}}+(p q-1) \delta_{2}}{(q-1) \frac{\delta_{1}-\delta_{2}}{1-\delta_{2}}+(p q-1)} \quad \text { if } \quad \delta_{1} \geq \delta_{2}, \\
& \frac{n}{2} \leq \frac{1+p \frac{1-\delta_{1}}{1-\delta_{2}}+(p q-1) \delta_{2}}{(p-1) \frac{\delta_{2}-\delta_{1}}{1-\delta_{1}}+(p q-1)} \quad \text { if } \quad \delta_{2} \geq \delta_{1},
\end{aligned}
$$

then there is no global (in time) Sobolev solution $(u, v) \in \mathcal{C}\left([0, \infty) \times \mathbb{L}^{2}\left(\mathbb{R}^{n}\right)\right) \times \mathcal{C}\left([0, \infty) \times \mathbb{L}^{2}\left(\mathbb{R}^{n}\right)\right)$ to (3). The critical exponent to the following structurally damped wave equation with the power nonlinearity $\left|u_{t}\right|^{p}$ :

$$
\left\{\begin{array}{l}
u_{t t}-\Delta u+\mu(-\Delta)^{\frac{\alpha}{2}} u_{t}=\left|u_{t}\right|^{p}, \quad x \in \mathbb{R}^{n}, \\
u(0, x)=u_{0}(x), \quad u_{t}(0, x)=u_{1}(x) \quad x \in \mathbb{R}^{n},
\end{array}\right.
$$

has been studied by Tuan Anh Dao and Ahmad Z. Fino in [11]. It was shown in [11] that if

$$
1<p \leq 1+\frac{\tilde{\alpha}}{n} \quad \text { where } \quad \tilde{\alpha}=\min \{1, \alpha\},
$$

then there is no global (in time) weak solution to (4). Note that one of the most typical important methods to verify critical exponent is well-known test function method ( see [13]). Concretely, this method is used to prove the nonexistence of global solutions by a contradiction argument. However, standard test function method seems difficult to be applied to $(1)$ containing pseudo-differential operators $(-\Delta)^{\frac{\alpha}{2}}$ for any $\alpha \in(0,2]$. The difficulty is caused by the nonlocal property of the fractional Laplacian operator. D' Abbicco and Reissig in [2] investigated the structurally damped wave equation with the power nonlinearity $|u|^{p}$. The critical exponent has been studied and they proposed to distinguish between (parabolic like models) in the case $\sigma \in(0,1]$, the so-called effective damping, and (hyperbolic like models) in the remaining case $\sigma \in(1,2]$, the so-called noneffective damping according to expected decay estimates (see more [3]). In the former case, they proved the existence of global (in time) solutions when

$$
p>p_{c}=1+\frac{2}{(n-\sigma)_{+}}
$$

for the small initial data and low space dimensions $2 \leq n \leq 4$ by using the energy estimates. Last years, the Moore-Gibson-Thompson (MGT) equation, a linearization of a model for wave propagation in viscous 
thermally relaxing fluids has been studied by several authors (see [14, [6], [7, [16], [8], [17] and references therein). This model is realized through the third order hyperbolic partial differential equation

$$
\tau u_{t t t}+u_{t t}-c^{2} \Delta u-b \Delta u_{t}=0,
$$

where the unknown function $u$ denotes a scalar acoustic velocity, $c$ denotes the speed of sound and $\tau$ denotes the thermal relaxation. Besides, the coefficient $b=\beta c^{2}$ is related to the diffusivity of the sound with $\tau \in(0, \beta]$. Let us underline that, to our knowledge, the MGT equation has not been widely investigated in the case of presence of non-local operators. For other contributions related to the semi-linear Moore-Gibson-Thompson equation with the power nonlinearity of derivative type we refer the reader to [7], [8] and references therein.

Motivated by the above contributions, our goal in this paper is to investigate problems (1) and (2). The paper is organized as follows. In the next section, we give some auxiliary results and formulate our main results. In Section 3, we prove our main results.

\section{Auxiliary Results}

Before to formulate our main results, we need the following definitions.

Definition 2.1. ([15],[18]) Let $s \in(0,1)$. Let $X$ be a suitable set of functions defined on $\mathbb{R}^{n}$. Then, the fractional Laplacian $(-\Delta)^{s}$ in $\mathbb{R}^{n}$ is a non-local operator given by

$$
(-\Delta)^{s}: f \in X \rightarrow(-\Delta)^{s} f(x)=C_{n, s} P . V \int_{\mathrm{IR}^{n}} \frac{f(x)-f(y)}{|x-y|^{n+2 s}} d y
$$

as long as the right-hand side exists. Here P.V stands for the Cauchy's principal value and $C_{n, s}=\frac{4^{s} \Gamma\left(\frac{n}{2}+s\right)}{\pi^{\frac{n}{2}} \Gamma(-s)}$ is the normalization constant and $\Gamma$ denotes the Gamma function.

Definition 2.2. (Weak solution for (1)) Let $T>0, p>1$, and $\left(u_{0}, u_{1}, u_{2}\right) \in H^{2}\left(\mathbb{R}^{n}\right) \times H^{1}\left(\mathbb{R}^{n}\right) \times$ $\mathbb{L}^{2}\left(\mathbb{R}^{n}\right)$. We say that $u \in \mathcal{C}\left([0, T), H^{2}\left(\mathbb{R}^{n}\right)\right) \cap \mathcal{C}^{1}\left([0, T), H^{1}\left(\mathbb{R}^{n}\right)\right) \cap \mathcal{C}^{2}\left([0, T), \mathbb{L}^{2}\left(\mathbb{R}^{n}\right)\right)$, satisfying $u_{t} \in$ $\mathbb{L}_{l o c}^{p}\left([0, T) \times \mathbb{R}^{n}\right)$, is a local weak solution to $(1)$ if

$$
\begin{aligned}
& \int_{0}^{T} \int_{\mathrm{IR}^{n}}\left|u_{t}(x, t)\right|^{p} \varphi(t, x) d x d t+\int_{\mathrm{IR}^{n}}\left(u_{1}(x)+u_{2}(x)\right) \varphi(0, x) d x \\
& -\int_{\mathrm{IR}^{n}} u_{1}(x) \varphi_{t}(0, x) d x=\int_{0}^{T} \int_{\mathbb{R}^{n}} u_{t}(x, t) \varphi_{t t}(t, x) d x d t \\
& -\int_{0}^{T} \int_{\mathrm{IR}^{n}} u_{t}(x, t) \varphi_{t}(t, x) d x d t-\int_{0}^{T} \int_{\mathrm{IR}^{n}} u_{t}(x, t)(-\Delta)^{\frac{\alpha}{2}} \varphi(x, t) d x d t \\
& -\int_{0}^{T} \int_{\mathrm{IR}^{n}} u(x, t) \Delta \varphi(x, t) d x d t
\end{aligned}
$$

for any test function $\varphi \in \mathcal{C}_{0}^{\infty}\left([0, T) \times \mathbb{R}^{n}\right)$ such that its support in time is compact and $\varphi(x, T)=\varphi_{t}(x, T)=$ $\varphi_{t t}(x, T)=0$ for all $x \in \mathbb{R}^{n}$. If $T=\infty$, we say that $u$ is a global weak solution to (1).

Definition 2.3. (Weak solution for (2)) Let $p, q>1$ and $T>0$. We say that $(u, v)$ is a local weak solution to the problem (2) if $\left(u_{t}, v_{t}\right) \in \mathbb{L}_{l o c}^{q}\left([0, T) \times \mathbb{R}^{n}\right) \times \mathbb{L}_{l o c}^{p}\left([0, T) \times \mathbb{R}^{n}\right)$ and satisfies the equations

$$
\begin{aligned}
& \int_{0}^{T} \int_{\mathbb{R}^{n}}\left|v_{t}(x, t)\right|^{p} \varphi(t, x) d x d t+\int_{\mathbb{R}^{n}}\left(u_{1}(x)+u_{2}(x)\right) \varphi(0, x) d x \\
& -\int_{\mathbb{I R}^{n}} u_{1}(x) \varphi_{t}(0, x) d x=\int_{0}^{T} \int_{\mathbb{R}^{n}} u_{t}(x, t) \varphi_{t t}(t, x) d x d t \\
& -\int_{0}^{T} \int_{\operatorname{IR}^{n}} u_{t}(x, t) \varphi_{t}(t, x) d x d t-\int_{0}^{T} \int_{\mathrm{IR}^{n}} u_{t}(x, t)(-\Delta)^{\frac{\alpha}{2}} \varphi(x, t) d x d t \\
& -\int_{0}^{T} \int_{\mathbb{I R}^{n}} u(x, t) \Delta \varphi(x, t) d x d t,
\end{aligned}
$$


and

$$
\begin{aligned}
& \int_{0}^{T} \int_{\mathrm{IR}^{n}}\left|u_{t}(x, t)\right|^{q} \varphi(t, x) d x d t+\int_{\mathrm{IR}^{n}}\left(v_{1}(x)+v_{2}(x)\right) \varphi(0, x) d x \\
& -\int_{\mathrm{IR}^{n}} v_{1}(x) \varphi_{t}(0, x) d x=\int_{0}^{T} \int_{\mathrm{IR}^{n}} v_{t}(x, t) \varphi_{t t}(t, x) d x d t \\
& -\int_{0}^{T} \int_{\mathrm{IR}^{n}} v_{t}(x, t) \varphi_{t}(t, x) d x d t-\int_{0}^{T} \int_{\mathbb{R}^{n}} v_{t}(x, t)(-\Delta)^{\frac{\beta}{2}} \varphi(x, t) d x d t \\
& -\int_{0}^{T} \int_{\mathrm{IR}^{n}} v(x, t) \Delta \varphi(x, t) d x d t
\end{aligned}
$$

for any test function $\varphi \in \mathcal{C}_{0}^{\infty}\left([0, T) \times \mathbb{R}^{n}\right)$ such that its support in time is compact and $\varphi(x, T)=\varphi_{t}(x, T)=$ $\varphi_{t t}(x, T)=0$ for all $x \in \mathbb{R}^{n}$. If $T=\infty$, we say that $(u, v)$ is a global weak solution to (2).

Now, we are ready to state the main results of this paper.

Theorem 2.4. Let $\alpha \in(0,2]$ and $\tilde{\alpha}=\min \{1, \alpha\}$. We assume that $\left(u_{0}, u_{1}, u_{2}\right) \in H^{2}\left(\mathbb{R}^{n}\right) \times H^{1}\left(\mathbb{R}^{n}\right) \times \mathbb{L}^{2}\left(\mathbb{R}^{n}\right)$ satisfy the following condition:

$$
\int_{\mathbb{R}^{n}}\left(u_{1}(x)+u_{2}(x)\right) \varphi(0, x) d x>0 .
$$

If

$$
1<p \leq 1+\frac{\tilde{\alpha}}{n}
$$

then there is no global (in time) weak solution to problem (1). Moreover, the sharp behavior of the lifespan $T_{\varepsilon}$ of local (in time) solutions to (1) with respect to a sufficiently small parameter $\varepsilon>0$ is given by

$$
T_{\varepsilon} \leq C \varepsilon^{-\frac{\tilde{\alpha}(p-1)}{\alpha-(p-1) n}}, \quad \text { for all small positive constant } \varepsilon \text {. }
$$

Theorem 2.5. Let $\alpha, \beta \in(0,2], \tilde{\alpha}=\min \{1, \alpha\}$, and $\tilde{\beta}=\min \{1, \beta\}$. We assume that $\left(u_{0}, u_{1}, u_{2}\right) \in$ $H^{2}\left(\mathbb{R}^{n}\right) \times H^{1}\left(\mathbb{R}^{n}\right) \times \mathbb{L}^{2}\left(\mathbb{R}^{n}\right)$ and $\left(v_{0}, v_{1}, v_{2}\right) \in H^{2}\left(\mathbb{R}^{n}\right) \times H^{1}\left(\mathbb{R}^{n}\right) \times \mathbb{L}^{2}\left(\mathbb{R}^{n}\right)$ satisfy the following conditions:

$$
\left\{\begin{array}{l}
\int_{\mathbb{R}^{n}}\left(u_{1}(x)+u_{2}(x)\right) \varphi(0, x) d x>0 \\
\int_{\mathbb{R}^{n}}\left(v_{1}(x)+v_{2}(x)\right) \varphi(0, x) d x>0 .
\end{array}\right.
$$

If

$$
n \leq \frac{1}{p q-1} \max \{\tilde{\beta}+\tilde{\alpha} p, \tilde{\alpha}+\tilde{\beta} q\}
$$

then there is no global (in time) weak solution to (2). Moreover, the blow-up time $T_{\varepsilon}$ is estimated by

$$
T_{\varepsilon} \leq C \varepsilon^{-\frac{\tilde{\alpha}}{p q-1}-n} \text { for all small positive constants } \varepsilon \text {. }
$$

The proofs of our main results are given in the next section. For the proofs of Theorems 2.4 and 2.5 , we shall use the nonlinear capacity method combined with the following pointwise estimate (see Dao and Reissig [12]).

Lemma 2.6. ([12] Let $\langle x\rangle=\left(1+(|x|-1)^{4}\right)^{\frac{1}{4}}$. Let $s \in(0,1)$ and $\phi: \mathbb{R}^{n} \rightarrow \mathbb{R}$ be the function defined by

$$
\phi(x)= \begin{cases}\langle x\rangle^{-n-2 s} & \text { if } \quad|x| \geq 1, \\ 1 & \text { if } \quad|x| \leq 1\end{cases}
$$


Then $\phi \in \mathcal{C}^{2}\left(\mathbb{R}^{n}\right)$, and the following estimate holds

$$
\left|(-\Delta)^{s} \phi(x)\right| \leq C \phi(x), x \in \mathbb{R}^{n},
$$

where $C$ is a constant independent of $x$.

Lemma 2.7. ([12]) Let $s \in(0,1)$. Let $\psi$ be a smooth function satisfying $\partial_{x}^{2} \psi \in \mathbb{L}^{\infty}\left(\mathbb{R}^{n}\right)$. For any $R>0$, let $\psi_{R}$ be a function defined by

$$
\psi_{R}(x)=\psi\left(\frac{x}{R}\right), \quad \text { for all } x \in \mathbb{R}^{n} .
$$

Then, $(-\Delta)^{s} \psi_{R}$ satisfies the following scaling properties:

$$
(-\Delta)^{s}\left(\psi_{R}\right)(x)=R^{-2 s}(-\Delta)^{s} \psi\left(\frac{x}{R}\right) \quad \text { for all } \quad x \in \mathbb{R}^{n} .
$$

Remark 2.8. Throughout, $C$ denotes a positive constant, whose value may change from line to line.

\subsection{Proof of Theorem 2.4}

Let $u$ be a global weak solution to $(1)$, then for all $\varphi \in \mathcal{C}\left([0, \infty) ; H^{2}\left(\mathbb{R}^{n}\right)\right) \cap \mathcal{C}^{1}\left([0, \infty) ; \mathbb{L}^{2}\left(\mathbb{R}^{n}\right)\right)$, one has

$$
\begin{aligned}
& \int_{0}^{+\infty} \int_{\mathbb{R}^{n}}\left|u_{t}(x, t)\right|^{p} \varphi(t, x) d x d t+\int_{\mathrm{IR}^{n}}\left(u_{1}(x)+u_{2}(x)\right) \varphi(0, x) d x \\
& -\int_{\mathrm{IR}^{n}} u_{1}(x) \varphi_{t}(0, x) d x=\int_{0}^{+\infty} \int_{\mathbb{R}^{n}} u_{t}(x, t) \varphi_{t t}(t, x) d x d t \\
& -\int_{0}^{+\infty} \int_{\mathrm{IR}^{n}} u_{t}(x, t) \varphi_{t}(t, x) d x d t-\int_{0}^{+\infty} \int_{\mathrm{IR}^{n}} u(x, t) \Delta \varphi(x, t) d x d t \\
& -\int_{0}^{+\infty} \int_{\mathrm{IR}^{n}} u_{t}(x, t)(-\Delta)^{\frac{\alpha}{2}} \varphi(x, t) d x d t .
\end{aligned}
$$

Now, we introduce the function $\phi=\phi(x)$, defined in (14) with $s=\frac{\alpha}{2}$, and the function $\eta=\eta(t)$ having the following properties:

1. $\eta \in \mathcal{C}_{0}^{\infty}([0, \infty)) \quad$ and $\begin{cases}1 & \text { if } \quad 0 \leq t \leq \frac{1}{2}, \\ \text { decreasing } & \text { if } \quad \frac{1}{2} \leq t \leq 1 \\ 0 & \text { if } t \geq 1 .\end{cases}$

2. $\eta^{-\frac{1}{p}}(t)\left(|\eta(t)|+\left|\eta^{\prime}(t)\right|+\left|\eta^{\prime \prime}(t)\right|\right) \leq C$ for any $t \in\left[\frac{1}{2}, 1\right]$.

Let $R$ be a large parameter in $[0, \infty)$. We define the following test function:

$$
\varphi_{R}(x, t)=\eta_{R}(t) \phi_{R}(x)
$$

where $\left.\eta_{R}(t)=\eta\left(R^{-\tilde{\alpha}} t\right)\right)$ and $\phi_{R}(x)=\phi\left(R^{-1} K^{-1} x\right)$ for some $K \geq 1$ which will be fixed later. Moreover, we check easily that $\operatorname{supp}(\eta) \subset\left[0, R^{\tilde{\alpha}}\right]$. We define the functionals

$$
I_{1}=\int_{0}^{+\infty} \int_{\mathrm{IR}^{n}}\left|u_{t}(x, t)\right|^{p} \varphi_{R}(t, x) d x d t=\int_{0}^{R^{\tilde{\alpha}}} \int_{\mathrm{IR}^{n}}\left|u_{t}(x, t)\right|^{p} \varphi_{R}(t, x) d x d t,
$$

and

$$
I_{2}=\int_{\frac{R^{\tilde{\alpha}}}{2}}^{R^{\tilde{\alpha}}} \int_{\mathbb{R}^{n}}\left|u_{t}(x, t)\right|^{p} \varphi_{R}(t, x) d x d t, \quad I_{3}=\int_{0}^{R^{\tilde{\alpha}}} \int_{\{|x| \geq R K\}}\left|u_{t}(x, t)\right|^{p} \varphi_{R}(t, x) d x d t .
$$


From $(16)$, one obtains

$$
\begin{aligned}
& I_{1}+\int_{\mathrm{IR}^{n}}\left(u_{1}(x)+u_{2}(x)\right) \phi_{R}(x) d x=\int_{\frac{R^{\tilde{\alpha}}}{2}}^{R^{\tilde{\alpha}}} \int_{\mathrm{IR}^{n}} u_{t}(x, t) \eta_{R}^{\prime \prime}(t) \phi_{R}(x) d x d t \\
& -\int_{\frac{R^{\tilde{\alpha}}}{2}}^{R^{\tilde{\alpha}}} \int_{\mathrm{IR}^{n}} u_{t}(x, t) \eta_{R}^{\prime}(t) \phi_{R}(x) d x d t-\int_{0}^{R^{\tilde{\alpha}}} \int_{\{|x| \geq R K\}} u(x, t) \eta_{R}(t) \Delta \phi_{R}(x) d x d t \\
& -\int_{0}^{R^{\tilde{\alpha}}} \int_{\mathrm{IR}^{n}} u_{t}(x, t) \eta_{R}(t)(-\Delta)^{\frac{\alpha}{2}} \phi_{R}(x) d x d t .
\end{aligned}
$$

Using integrating by parts, one has

$$
\begin{aligned}
& I_{1}+\int_{\mathbb{R}^{n}}\left(u_{1}(x)+u_{2}(x)\right) \phi_{R}(x) d x+\int_{\mathbb{R}^{n}} u_{0}(x) \Psi_{R}(0) \Delta \phi_{R}(x) d x \\
& =\int_{\frac{R^{\tilde{\alpha}}}{2}}^{R^{\tilde{\alpha}}} \int_{\mathbb{R}^{n}} u_{t}(x, t) \eta_{R}^{\prime \prime}(t) \phi_{R}(x) d x d t \\
& -\int_{\frac{R^{\tilde{\alpha}}}{2}}^{R^{\tilde{\alpha}}} \int_{\mathbb{I R}^{n}} u_{t}(x, t) \eta_{R}^{\prime}(t) \phi_{R}(x) d x d t \\
& +\int_{0}^{R^{\tilde{\alpha}}} \int_{\{|x| \geq R K\}} u_{t}(x, t) \Psi_{R}(t) \Delta \phi_{R}(x) d x d t \\
& -\int_{0}^{R^{\tilde{\alpha}}} \int_{\mathbb{R}^{n}} u_{t}(x, t) \eta_{R}(t)(-\Delta)^{\frac{\alpha}{2}} \phi_{R}(x) d x d t=J_{1}-J_{2}+J_{3}-J_{4},
\end{aligned}
$$

where

$$
\Psi_{R}(t)=\int_{t}^{R^{\tilde{\alpha}}} \eta_{R}(\tau) d \tau
$$

Applying Hölder's inequality with $\frac{1}{p}+\frac{1}{p^{\prime}}=1$, we can proceed the estimate for $J_{1}$ as follows:

$$
\begin{aligned}
\left|J_{1}\right| & \leq C \int_{\frac{R^{\tilde{\alpha}}}{2}}^{R^{\tilde{\alpha}}} \int_{\mathrm{IR}^{n}}\left|u_{t}(x, t)\right|\left|\eta_{R}^{\prime \prime}(t)\right| \phi_{R}(x) d x d t \\
& \left.\leq\left(\int_{\frac{R^{\tilde{\alpha}}}{2}}^{R^{\tilde{\alpha}}} \int_{\mathbb{R}^{n}}\left(\left|u_{t}(x, t)\right| \varphi_{R}^{\frac{1}{p}}(t, x)\right)\right)^{p} d x d t\right)^{\frac{1}{p}} \\
& \times\left(\int_{\frac{R^{\tilde{\alpha}}}{2}}^{R^{\tilde{\alpha}}} \int_{\mathbb{R}^{n}}\left(\left|\eta_{R}^{\prime \prime}(t)\right| \phi_{R}(x) \varphi_{R}^{-\frac{1}{p}}(t, x)\right)^{p^{\prime}} d x d t\right)^{\frac{1}{p^{\prime}}} \\
& \leq C I_{2}^{\frac{1}{p}}\left(\int_{\frac{R^{\tilde{\alpha}}}{2}}^{R^{\tilde{\alpha}}} \int_{\mathbb{I R}^{n}} \eta_{R}^{-\frac{p^{\prime}}{p}}(t)\left|\eta_{R}^{\prime \prime}(t)\right|^{p^{\prime}} \phi_{R}(x) d x d t\right)^{\frac{1}{p^{\prime}}} .
\end{aligned}
$$

Using change of variables $\tilde{t}=R^{-\tilde{\alpha}} t$ and $\tilde{x}=R^{-1} K^{-1} x$, we get

$$
\left|J_{1}\right| \leq C I_{2}^{\frac{1}{p}} R^{-2 \tilde{\alpha}+\frac{n+\tilde{\alpha}}{p^{\prime}}} K^{\frac{n}{p^{\prime}}}\left(\int_{\operatorname{IR}^{n}}\langle\tilde{x}\rangle^{-n-\alpha} d \tilde{x}\right)^{\frac{1}{p^{\prime}}} \leq C I_{2}^{\frac{1}{p}} R^{-2 \tilde{\alpha}+\frac{n+\tilde{\alpha}}{p^{\prime}}} K^{\frac{n}{p^{\prime}}}
$$

Now, let us turn to estimate $J_{2}, J_{3}$, and $J_{4}$. Applying Hölder 's inequality again, as we estimated $J_{1}$, leads to

$$
\begin{aligned}
\left|J_{3}\right| & \leq C I_{3}^{\frac{1}{p}}\left(\int_{0}^{R^{\tilde{\alpha}}} \int_{\{|x| \geq R K\}} \Psi_{R}^{p^{\prime}}(t) \eta_{R}^{-\frac{p^{\prime}}{p}}(t) \phi_{R}^{-\frac{p^{\prime}}{p}}(x)\left|\Delta \phi_{R}(x)\right|^{p^{\prime}} d x d t\right)^{\frac{1}{p^{\prime}}} \\
& \leq C I_{3}^{\frac{1}{p}} R^{-2+\tilde{\alpha}+\frac{n+\tilde{\alpha}}{p^{\prime}}} K^{-2+\frac{n}{p^{\prime}}},
\end{aligned}
$$




$$
\begin{aligned}
\left|J_{2}\right| & \leq C I_{2}^{\frac{1}{p}}\left(\int_{\frac{R^{\tilde{\alpha}}}{2}}^{R^{\tilde{\alpha}}} \int_{\mathrm{IR}^{n}} \eta_{R}^{-\frac{p^{\prime}}{p}}(t)\left|\eta_{R}^{\prime}(t)\right|^{p^{\prime}} \phi_{R}(x) d x d t\right)^{\frac{1}{p^{\prime}}} \\
& \leq C I_{2}^{\frac{1}{p}} R^{-\tilde{\alpha}+\frac{n+\tilde{\alpha}}{p^{\prime}}} K^{\frac{n}{p^{\prime}}}\left(\int_{\mathrm{IR}^{n}}\langle\tilde{x}\rangle^{-n-\alpha} d \tilde{x}\right)^{\frac{1}{p^{\prime}}} \leq C I_{2}^{\frac{1}{p}} R^{-\tilde{\alpha}+\frac{n+\tilde{\alpha}}{p^{\prime}}} K^{\frac{n}{p^{\prime}}},
\end{aligned}
$$

and

$$
\begin{aligned}
\left|J_{4}\right| & \leq C I_{1}^{\frac{1}{p}}\left(\int_{0}^{R^{\tilde{\alpha}}} \int_{\mathrm{IR}^{n}} \eta_{R}(t) \phi_{R}^{-\frac{p^{\prime}}{p}}(x)\left|(-\Delta)^{\frac{\alpha}{2}} \phi_{R}(x)\right|^{p^{\prime}} d x d t\right)^{\frac{1}{p^{\prime}}} \\
& \leq C I_{1}^{\frac{1}{p}} R^{-\alpha+\frac{n+\tilde{\alpha}}{p^{\prime}}} K^{-\alpha+\frac{n}{p^{\prime}}} .
\end{aligned}
$$

Combining the estimates from 18 to 21 we may arrive at

$$
\begin{aligned}
I_{1} & +\int_{\mathbb{R}^{n}}\left(u_{1}(x)+u_{2}(x)\right) \phi_{R}(x) d x \leq \int_{\mathrm{IR}^{n}}\left|u_{0}(x)\left\|\Psi_{R}(0)\right\| \Delta \phi_{R}(x)\right| d x \\
& +C\left(I_{2}^{\frac{1}{p}} R^{-2 \tilde{\alpha}+\frac{n+\tilde{\alpha}}{p^{\prime}}} K^{\frac{n}{p^{\prime}}}+I_{2}^{\frac{1}{p}} R^{-\tilde{\alpha}+\frac{n+\tilde{\alpha}}{p^{\prime}}} K^{\frac{n}{p^{\prime}}}+I_{3}^{\frac{1}{p}} R^{-2+\tilde{\alpha}+\frac{n+\tilde{\alpha}}{p^{\prime}}} K^{-2+\frac{n}{p^{\prime}}}\right. \\
& \left.+I_{1}^{\frac{1}{p}} R^{-\alpha+\frac{n+\tilde{\alpha}}{p^{\prime}}} K^{-\alpha+\frac{n}{p^{\prime}}}\right) .
\end{aligned}
$$

Moreover, it is clear that

$$
\Psi_{R}(t)=\int_{t}^{R^{\tilde{\alpha}}} \eta_{R}(\tau) d \tau=R^{\tilde{\alpha}}-t \quad \text { then } \quad \Psi_{R}(0)=R^{\tilde{\alpha}} .
$$

We can easily check that $\left|\Delta \phi_{R}(x)\right| \leq R^{-2} \phi_{R}(x)$. Therefore, this implies that

$$
\begin{aligned}
& I_{1}+\int_{\mathrm{IR}^{n}}\left(u_{1}(x)+u_{2}(x)\right) \phi_{R}(x) d x \leq R^{\tilde{\alpha}-2} \int_{\mathrm{IR}^{n}}\left|u_{0}(x)\right| \phi_{R}(x) d x \\
& +C\left(I_{2}^{\frac{1}{p}} R^{-2 \tilde{\alpha}+\frac{n+\tilde{\alpha}}{p^{\prime}}} K^{\frac{n}{p^{\prime}}}+I_{2}^{\frac{1}{p}} R^{-\tilde{\alpha}+\frac{n+\tilde{\alpha}}{p^{\prime}}} K^{\frac{n}{p^{\prime}}}+I_{3}^{\frac{1}{p}} R^{-2+\tilde{\alpha}+\frac{n+\tilde{\alpha}}{p^{\prime}}} K^{-2+\frac{n}{p^{\prime}}}\right. \\
& \left.+I_{1}^{\frac{1}{p}} R^{-\alpha+\frac{n+\tilde{\alpha}}{p^{\prime}}} K^{-\alpha+\frac{n}{p^{\prime}}}\right) .
\end{aligned}
$$

Since $u_{0} \in \mathbb{L}^{1}\left(\mathbb{R}^{n}\right)$, it implies immediately that

$$
\lim _{R \rightarrow \infty}\left[R^{\tilde{\alpha}-2} \int_{\mathrm{IR}^{n}}\left|u_{0}(x)\right| \phi_{R}(x) d x\right]=0 .
$$

Invoking the assumption (8), one obtains

$$
R^{\tilde{\alpha}-2} \int_{\mathbb{R}^{n}}\left|u_{0}(x)\right| \phi_{R}(x) d x<\frac{1}{2} \int_{\mathbb{R}^{n}}\left(u_{1}(x)+u_{2}(x)\right) \phi_{R}(x) d x .
$$

From (22), we easily see that

$$
\begin{aligned}
& I_{1}+\frac{1}{2} \int_{\mathrm{IR}^{n}}\left(u_{1}(x)+u_{2}(x)\right) \phi_{R}(x) d x \leq C\left(I_{2}^{\frac{1}{p}} R^{-2 \tilde{\alpha}+\frac{n+\tilde{\alpha}}{p^{\prime}}} K^{\frac{n}{p^{\prime}}}\right. \\
& \left.+I_{2}^{\frac{1}{p}} R^{-\tilde{\alpha}+\frac{n+\tilde{\alpha}}{p^{\prime}}} K^{\frac{n}{p^{\prime}}}+I_{3}^{\frac{1}{p}} R^{-2+\tilde{\alpha}+\frac{n+\tilde{\alpha}}{p^{\prime}}} K^{-2+\frac{n}{p^{\prime}}}+I_{1}^{\frac{1}{p}} R^{-\alpha+\frac{n+\tilde{\alpha}}{p^{\prime}}} K^{-\alpha+\frac{n}{p^{\prime}}}\right) .
\end{aligned}
$$


By choosing $K=1$ and noticing the relations $I_{2} \leq I_{1}$ and $I_{3} \leq I_{1}$, we may arrive at

$$
\begin{aligned}
& I_{1}+\frac{1}{2} \int_{\mathrm{IR}^{n}}\left(u_{1}(x)+u_{2}(x)\right) \phi_{R}(x) d x \leq C\left(I_{1}^{\frac{1}{p}} R^{-2 \tilde{\alpha}+\frac{n+\tilde{\alpha}}{p^{\prime}}}\right. \\
& \left.+I_{1}^{\frac{1}{p}} R^{-\tilde{\alpha}+\frac{n+\tilde{\alpha}}{p^{\prime}}}+I_{1}^{\frac{1}{p}} R^{-2+\tilde{\alpha}+\frac{n+\tilde{\alpha}}{p^{\prime}}}+I_{1}^{\frac{1}{p}} R^{-\alpha+\frac{n+\tilde{\alpha}}{p^{\prime}}}\right) \leq C I_{1}^{\frac{1}{p}} R^{-\tilde{\alpha}+\frac{n+\tilde{\alpha}}{p^{\prime}}} .
\end{aligned}
$$

Thanks to the following $\varepsilon$-Young's inequality:

$$
a b \leq \varepsilon a^{p}+C(\varepsilon) b^{p^{\prime}}, \quad \text { for all } a, b>0 \text { and for any } \varepsilon>0,
$$

we conclude

$$
C I_{1}^{\frac{1}{p}} R^{-\tilde{\alpha}+\frac{n+\tilde{\alpha}}{p^{\prime}}} \leq \varepsilon I_{1}+C(\varepsilon) R^{-\tilde{\alpha} p^{\prime}+n+\tilde{\alpha}} .
$$

Consequently, from 24) we derive

$$
(1-\varepsilon) I_{1}+\frac{1}{2} \int_{\mathbb{R}^{n}}\left(u_{1}(x)+u_{2}(x)\right) \phi_{R}(x) d x \leq C(\varepsilon) R^{-\tilde{\alpha} p^{\prime}+n+\tilde{\alpha}},
$$

which follows that

$$
I_{1} \leq C R^{-\tilde{\alpha} p^{\prime}+n+\tilde{\alpha}}
$$

and

$$
\int_{\mathbb{R}^{n}}\left(u_{1}(x)+u_{2}(x)\right) \phi_{R}(x) d x \leq C R^{-\tilde{\alpha} p^{\prime}+n+\tilde{\alpha}} .
$$

It is clear that the assumption $(9)$ is equivalent to $-\tilde{\alpha} p^{\prime}+n+\tilde{\alpha} \leq 0$. For this reason, we will split our consideration into two cases.

Case 1:In the subcritical case $-\tilde{\alpha} p^{\prime}+n+\tilde{\alpha}<0$, letting $R \rightarrow \infty$ in $(26)$, we easily deduce

$$
\int_{\mathbb{R}^{n}}\left(u_{1}(x)+u_{2}(x)\right) \phi_{R}(x) d x \leq 0
$$

which contradicts the assumption (8).

Case 2: For the critical case $-\tilde{\alpha} p^{\prime}+n+\tilde{\alpha}=0$, from (25), we can see that $I_{1} \leq C$. Using Beppo Levi's theorem on monotone convergence, one obtains

$$
\begin{aligned}
\int_{0}^{\infty} \int_{\mathbb{I R}^{n}}\left|u_{t}(x, t)\right|^{p} d x d t & =\lim _{R \rightarrow \infty} \int_{0}^{R^{\tilde{\alpha}}} \int_{\mathbb{R}^{n}}\left|u_{t}(x, t)\right|^{p} \varphi_{R}(x, t) d x d t \\
& =\lim _{R \rightarrow \infty} I_{1} \leq C .
\end{aligned}
$$

We conclude that $u_{t} \in \mathbb{L}^{p}\left((0, \infty) \times \mathbb{R}^{n}\right)$. By the absolute continuity of the Lebesgue integral, it follows that $I_{2} \rightarrow 0$ and $I_{3} \rightarrow 0$, as $R \rightarrow \infty$. Using again the fact that $\tilde{\alpha}=\frac{n+\tilde{\alpha}}{p^{\prime}}$, we obtain from $\sqrt{23}$ the following estimate:

$$
\begin{aligned}
I_{1} & +\frac{1}{2} \int_{\mathrm{IR}^{n}}\left(u_{1}(x)+u_{2}(x)\right) \phi_{R}(x) d x \leq C\left(I_{2}^{\frac{1}{p}} R^{-\tilde{\alpha}} K^{\frac{n}{p^{\prime}}}+I_{2}^{\frac{1}{p}} K^{\frac{n}{p^{\prime}}}\right. \\
& \left.+I_{3}^{\frac{1}{p}} R^{-2+2 \tilde{\alpha}} K^{-2+\frac{n}{p^{\prime}}}+I_{1}^{\frac{1}{p}} R^{-\alpha+\tilde{\alpha}} K^{-\alpha+\frac{n}{p^{\prime}}}\right),
\end{aligned}
$$

for all $K \geq 1$.

1. If $\alpha \in(0,1]$, then $\alpha=\tilde{\alpha}$. Consequently, from (27), we have

$$
\begin{aligned}
I_{1} & +\frac{1}{2} \int_{\mathrm{IR}^{n}}\left(u_{1}(x)+u_{2}(x)\right) \phi_{R}(x) d x \leq C\left(I_{2}^{\frac{1}{p}} R^{-\alpha} K^{\frac{n}{p^{\prime}}}+I_{2}^{\frac{1}{p}} K^{\frac{n}{p^{\prime}}}\right. \\
& \left.+I_{3}^{\frac{1}{p}} R^{-2(1-\alpha)} K^{-2+\frac{n}{p^{\prime}}}+I_{1}^{\frac{1}{p}} K^{-\alpha+\frac{n}{p^{\prime}}}\right) .
\end{aligned}
$$


Letting $R \rightarrow \infty$ in 28 , we get

$$
\int_{\mathrm{IR}^{n}}\left(u_{1}(x)+u_{2}(x)\right) \phi_{R}(x) d x \leq C K^{-\alpha+\frac{n}{p^{\prime}}} \text { for all } \quad K \geq 1 .
$$

It is obvious that $-\alpha+\frac{n}{p^{\prime}}<0$. We can fix a sufficiently large constant $K \geq 1$ in 29 to gain a contradiction to 8 .

2. If $\alpha \in(1,2]$, then $\tilde{\alpha}=1$. As a result, choosing $K=1$, we may conclude from (27) that

$$
I_{1}+\frac{1}{2} \int_{\mathrm{IR}^{n}}\left(u_{1}(x)+u_{2}(x)\right) \phi_{R}(x) d x \leq C\left(I_{2}^{\frac{1}{p}} R^{-1}+I_{2}^{\frac{1}{p}}+I_{3}^{\frac{1}{p}}+I_{1}^{\frac{1}{p}} R^{1-\alpha}\right) .
$$

Since $\alpha>1$, letting $R \rightarrow \infty$ in (30) we obtain a contradiction to (8) again.

Let us now consider the case of subcritical exponent to prove the estimate for lifespan $T_{\varepsilon}$ of solutions to (1). We assume that $u=u(x, t)$ is a local solution to (1). In order to prove the lifespan estimate, we replace the initial data $\left(0, u_{1}, u_{2}\right)$ by $\left(0, \varepsilon f_{1}, \varepsilon f_{2}\right)$ with a small constant $\varepsilon>0$, where $\left(f_{1}, f_{2}\right) \in H^{1}\left(\mathbb{R}^{n}\right) \times \mathbb{L}^{2}\left(\mathbb{R}^{n}\right)$ satisfy the assumption (8). Invoking the fact that

$$
\int_{\mathbb{R}^{n}}\left(f_{1}(x)+f_{2}(x)\right) \phi_{R}(x) d x \geq c>0,
$$

and repeating the steps in the above proofs we arrive at the following estimate:

$$
\varepsilon \leq C R^{-\tilde{\alpha} p^{\prime}+n+\tilde{\alpha}} .
$$

Let $R=T^{\frac{1}{\tilde{\alpha}}}$, then a standard calculation lead to

$$
T_{\varepsilon} \leq \varepsilon^{-\frac{\tilde{\alpha}(p-1)}{\tilde{\alpha}-(p-1) n}} .
$$

Summarizing, the proof of the Theorem 2.4 is completed.

\subsection{Proof of Theorem 2.5}

First, we introduce the same test function as in the proof of Theorem 2.4. Let us assume that $(u, v)$ is the global weak solution to (2). We define the functionals

$$
J_{1}=\int_{0}^{+\infty} \int_{\mathrm{IR}^{n}}\left|u_{t}(x, t)\right|^{q} \varphi_{R}(t, x) d x d t=\int_{0}^{R^{\tilde{\alpha}}} \int_{\mathrm{IR}^{n}}\left|u_{t}(x, t)\right|^{q} \varphi_{R}(t, x) d x d t,
$$

and

and

$$
\begin{gathered}
J_{2}=\int_{\frac{R^{\tilde{\alpha}}}{2}}^{R^{\tilde{\alpha}}} \int_{\mathbb{I R}^{n}}\left|u_{t}(x, t)\right|^{q} \varphi_{R}(t, x) d x d t, \quad J_{3}=\int_{0}^{R^{\tilde{\alpha}}} \int_{\{|x| \geq R K\}}\left|u_{t}(x, t)\right|^{q} \varphi_{R}(t, x) d x d t, \\
I_{1}=\int_{0}^{+\infty} \int_{\mathbb{R}^{n}}\left|v_{t}(x, t)\right|^{p} \varphi_{R}(t, x) d x d t=\int_{0}^{R^{\tilde{\alpha}}} \int_{\mathbb{R}^{n}}\left|v_{t}(x, t)\right|^{p} \varphi_{R}(t, x) d x d t,
\end{gathered}
$$

$$
I_{2}=\int_{\frac{R^{\tilde{\alpha}}}{2}}^{R^{\tilde{\alpha}}} \int_{\mathrm{IR}^{n}}\left|v_{t}(x, t)\right|^{p} \varphi_{R}(t, x) d x d t, \quad I_{3}=\int_{0}^{R^{\tilde{\alpha}}} \int_{\{|x| \geq R K\}}\left|v_{t}(x, t)\right|^{p} \varphi_{R}(t, x) d x d t .
$$

From (6) and (7), one has

$$
\begin{aligned}
& I_{1}+\int_{\mathrm{IR}^{n}}\left(u_{1}(x)+u_{2}(x)\right) \varphi(0, x) d x=\int_{\frac{R^{\tilde{\alpha}}}{2}}^{R^{\tilde{\alpha}}} \int_{\mathrm{IR}^{n}} u_{t}(x, t) \eta_{R}^{\prime \prime}(t) \phi_{R}(x) d x d t \\
& -\int_{\frac{R^{\tilde{\alpha}}}{2}}^{R^{\tilde{\alpha}}} \int_{\mathrm{IR}^{n}} u_{t}(x, t) \eta_{R}^{\prime}(t) \phi_{R}(x) d x d t-\int_{0}^{R^{\tilde{\alpha}}} \int_{\{|x| \geq R K\}} u(x, t) \eta_{R}(t) \Delta \phi_{R}(x) d x d t \\
& -\int_{0}^{R^{\tilde{\alpha}}} \int_{\mathrm{IR}^{n}} u_{t}(x, t) \eta_{R}(t)(-\Delta)^{\frac{\alpha}{2}} \phi_{R}(x) d x d t,
\end{aligned}
$$


and

$$
\begin{aligned}
& J_{1}+\int_{\mathbb{I}^{n}}\left(v_{1}(x)+v_{2}(x)\right) \varphi(0, x) d x=\int_{\frac{R^{\tilde{\alpha}}}{2}}^{R^{\tilde{\alpha}}} \int_{\mathbb{R}^{n}} v_{t}(x, t) \eta_{R}^{\prime \prime}(t) \phi_{R}(x) d x d t \\
& -\int_{\frac{R^{\tilde{\alpha}}}{2}}^{R^{\tilde{\alpha}}} \int_{\mathbb{R}^{n}} v_{t}(x, t) \eta_{R}^{\prime}(t) \phi_{R}(x) d x d t-\int_{0}^{R^{\tilde{\alpha}}} \int_{\{|x| \geq R K\}} v(x, t) \eta_{R}(t) \Delta \phi_{R}(x) d x d t \\
& -\int_{0}^{R^{\tilde{\alpha}}} \int_{\mathbb{R}^{n}} v_{t}(x, t) \eta_{R}(t)(-\Delta)^{\frac{\beta}{2}} \phi_{R}(x) d x d t .
\end{aligned}
$$

Repeating the steps of the proof from (18) to 24, we may conclude the following estimates:

$$
I_{1} \leq J_{1}^{\frac{1}{q}} R^{-\tilde{\alpha}+\frac{n+\tilde{\alpha}}{q^{\prime}}}
$$

In the analogous way, one obtains

$$
J_{1} \leq I_{1}^{\frac{1}{p}} R^{-\tilde{\beta}+\frac{n+\tilde{\beta}}{p^{\prime}}}
$$

From 31 and 32 , we obtain

$$
\begin{aligned}
& I_{1}^{\frac{p q-1}{p q}} \leq R^{\left(-\tilde{\beta}+\frac{n+\tilde{\beta}}{p^{\prime}}\right) \frac{1}{q}-\tilde{\alpha}+\frac{n+\tilde{\alpha}}{q^{\prime}}}=R^{\delta_{1}}, \\
& J_{1}^{\frac{p q-1}{p q}} \leq R^{\left(-\tilde{\alpha}+\frac{n+\tilde{\alpha}}{q^{\prime}}\right) \frac{1}{p}-\tilde{\beta}+\frac{n+\tilde{\beta}}{p^{\prime}}}=R^{\delta_{2}} .
\end{aligned}
$$

It is clear that the assumption $(12)$ is equivalent to $\max \left\{\delta_{1}, \delta_{2}\right\} \leq 0$. For this reason, we will split our consideration into two cases.

Case 1:In the subcritical case $\max \left\{\delta_{1}, \delta_{2}\right\}<0$, letting $R \rightarrow \infty$ in 33) and (34) we easily deduce

$$
\int_{\mathbb{R}^{n}}\left(v_{1}(x)+v_{2}(x)\right) \phi_{R}(x) d x \leq 0 \quad \text { and } \quad \int_{\mathbb{R}^{n}}\left(u_{1}(x)+u_{2}(x)\right) \phi_{R}(x) d x \leq 0,
$$

which contradicts the assumption (11).

Case 2: For the critical case $\delta_{2}=0$, from 25 we can see that $J_{1} \leq C$. Using Beppo Levi's theorem on monotone convergence, one obtains

$$
\begin{aligned}
\int_{0}^{\infty} \int_{\mathbb{R}^{n}}\left|u_{t}(x, t)\right|^{q} d x d t & =\lim _{R \rightarrow \infty} \int_{0}^{R^{\tilde{\alpha}}} \int_{\mathbb{R}^{n}}\left|u_{t}(x, t)\right|^{q} \varphi_{R}(x, t) d x d t \\
& =\lim _{R \rightarrow \infty} J_{1} \leq C .
\end{aligned}
$$

Repeating the steps of the proof from $(22)$ to $(24)$, we may conclude the following estimates:

$$
J_{1}+\frac{1}{2} \int_{\mathbb{R}^{n}}\left(v_{1}(x)+v_{2}(x)\right) \phi_{R}(x) d x \leq C\left(\int_{0}^{R^{\tilde{\alpha}}} \int_{\mathbb{R}^{n}}\left|v_{t}(x, t)\right|^{p} \varphi_{R}(t, x) d x d t\right)^{\frac{1}{p}} R^{-\tilde{\beta}+\frac{n+\tilde{\beta}}{p^{\prime}}}
$$

and

$$
I_{1}+\frac{1}{2} \int_{\mathrm{IR}^{n}}\left(u_{1}(x)+u_{2}(x)\right) \phi_{R}(x) d x \leq C\left(\int_{0}^{R^{\tilde{\alpha}}} \int_{\mathrm{IR}^{n}}\left|u_{t}(x, t)\right|^{q} \varphi_{R}(t, x) d x d t\right)^{\frac{1}{q}} R^{-\tilde{\alpha}+\frac{n+\tilde{\alpha}}{q^{\prime}}} .
$$

Since $\delta_{2}=0$ and invoking the above estimates, we easily deduce that

$$
\begin{aligned}
J_{1} & +\frac{1}{2} \int_{\mathrm{IR}^{n}}\left(v_{1}(x)+v_{2}(x)\right) \phi_{R}(x) d x \\
& \leq\left(\int_{0}^{R^{\tilde{\alpha}}} \int_{\mathrm{IR}^{n}}\left|u_{t}(x, t)\right|^{q} \varphi_{R}(t, x) d x d t\right)^{\frac{1}{p q}} .
\end{aligned}
$$


Letting $R \rightarrow \infty$ in 36 and using $(37)$, one obtains

$$
\int_{0}^{+\infty} \int_{\mathbb{R}^{n}}\left|u_{t}(x, t)\right|^{q} d x d t+\int_{\mathbb{R}^{n}}\left(v_{1}(x)+v_{2}(x)\right) \phi_{R}(x) d x=0,
$$

which is a contradiction to (11). In the case $\delta_{1}=0$ we repeat the same arguments as in $\delta_{2}=0$.

Let us now consider the case of subcritical exponent to prove the estimate for lifespan $T_{\varepsilon}$ of solutions to (2). We assume that $(u, v)=(u(x, t), v(x, t))$, is a local solution to (2). In order to prove the lifespan estimate, we replace the initial data $\left(0, u_{1}, u_{2}\right),\left(0, v_{1}, v_{2}\right)$ by $\left(0, \varepsilon f_{1}, \varepsilon f_{2}\right),\left(0, \varepsilon g_{1}, \varepsilon g_{2}\right)$ with a small constant $\varepsilon>0$, where $\left(f_{1}, f_{2}\right),\left(g_{1}, g_{2}\right) \in H^{1}\left(\mathbb{R}^{n}\right) \times \mathbb{L}^{2}\left(\mathbb{R}^{n}\right)$ satisfy the assumption 11$)$. Repeating the steps in the above proofs, we arrive at the following estimate:

$$
I_{1}+c \varepsilon \leq J_{1}^{\frac{1}{q}} R^{-\tilde{\alpha}+\frac{n+\tilde{\alpha}}{q^{\prime}}}
$$

and

$$
J_{1}+c \varepsilon \leq I_{1}^{\frac{1}{p}} R^{-\tilde{\beta}+\frac{n+\tilde{\beta}}{p^{\prime}}}
$$

If we plug 37 in 38$)$, we find

$$
J_{1}+c \varepsilon \leq C J_{1}^{\frac{1}{p q}} R^{\left(-\tilde{\beta}+\frac{n+\tilde{\beta}}{p^{\prime}}\right)+\left(-\tilde{\alpha}+\frac{n+\tilde{\alpha}}{q^{\prime}}\right) \frac{1}{p}}
$$

We easily obtains that

$$
c \varepsilon \leq C J_{1}^{\frac{1}{p q}} R\left(-\tilde{\beta}+\frac{n+\tilde{\beta}}{p^{\prime}}\right)+\left(-\tilde{\alpha}+\frac{n+\tilde{\alpha}}{q^{\prime}}\right) \frac{1}{p}-J_{1},
$$

which leads to

$$
\varepsilon \leq C R^{-\left[\frac{\tilde{\alpha}+\tilde{\beta} q}{p q-1}-n\right]} .
$$

Let $R=T^{\frac{1}{\tilde{\alpha}}}$. Then with a standard calculation, one has

$$
T_{\varepsilon} \leq \varepsilon^{-\frac{\tilde{\alpha}(p q-1)}{\tilde{\alpha}+\tilde{\beta} q-n(p q-1)}} .
$$

Summarizing, the proof of the Theorem 2.5 is completed.

\section{References}

[1] M. D'Abbicco, A note on a weakly coupled system of structurally damped waves, Dynamical Systems, Differential Equations and Applications, AIMS Proceedings. (2015) 320-329.

[2] M. D'Abbicco, M. Reissig, Semi-linear structural damped waves, Math. Methods Appl. Sci. 37 (2014) $1570-1592$.

[3] M. D'Abbicco, M.R. Ebert, A classifiation of structural dissipations for evolution operators, Math. Methods Appl. Sci. 39 (2016) 2558-2582.

[4] M. D'Abbicco, M.R. Ebert, A new phenomenon in the critical exponent for structurally damped semi-linear evolution equations, Nonlinear Anal. 149 (2017) 1-40.

[5] L. Caffarelli, L. Silvestre, An extension problem related to the fractional Laplacian, Comm. Partial Differential Equations. 32 (2007) 1245-1260.

[6] W. Chen, R. Ikehata, The Cauchy problem for the Moore-Gibson-Thompson equation in the dissipative case, J. Differential Equations. 292 (2021) 176-219.

[7] W. Chen, A. Palmieri, A blow-up result for the semilinear Moore-Gibson-Thompson equation with nonlinearity of derivative type in the conservative case, Evol. Equ. Control Theory. (2020) 1-15. DOI: 10.3934/eect.2020085.

[8] W. Chen, A. Palmieri, Nonexistence of global solutions for the semi-linear Moore-Gibson-Thompson equation in the conservative case, Discrete Contin. Dyn. Syst. 40(9) (2020) 5513-5540.

[9] T.A. Dao, Global existence of solutions for weakly coupled systems of semi-linear structurally damped $\sigma-$ evolution models with different power nonlinearities, 30A4, submitted.

[10] T.A. Dao, Existence and nonexistence of global solutions for a structurally damped wave system with power nonlinearities,arXiv: 1911.04412v1, 2019.

[11] T.A Dao, Ahmad Z. Fino, Critical exponent for semi-linear structurally damped wave equation of derivative type, arXiv: 2004.08486v2, 2020. 
[12] T.A. Dao, M. Reissig, A blow-up result for semi-linear structurally damped $\frac{\sigma}{2}$-evolution equations, preprint on arXiv:1909.01181v1, 2019.

[13] T. Hadj Kaddour, A. Hakem Suffucient conditions of non global solution for fractional damped wave equa- tions with non-linear memory, Advances in the Theory of Nonlinear Analysis and its Applications. 2(4) (2018) 224-237.

[14] B. Kaltenbacher, I. Lasiecka, Exponential decay for low and higher energies in the third order linear Moore-GibsonThompson equation with variable viscosity, Palest. J. Math. 1 (1) (2012) 1-10.

[15] M. Kwasnicki, Ten equivalent definitions of the fractional Laplace operator, Frac.Calc.Appl.Anal. 20(2017) 7-51.

[16] M. Pellicer, B. Said-Houari, Wellposedness and decay rates for the Cauchy problem of the Moore-Gibson-Thompson equation arising in high intensity ultrasound, Appl. Math. Optim. 80(2) (2019) 447-478.

[17] R. Racke, B. Said-Houari, Global well-posedness of the Cauchy problem for the 3D Jordan- Moore-Gibson-Thompson equation, Commun. Contemp. Math., in press, (2021).

[18] L. Silvestre, Regularity of the obstacle problem for a fractional power of the Laplace operator, Comm. Pure Appl. Math. 60 (1) (2007) 67-112. 